FACTA UNIVERSITATIS

Series: Linguistics and Literature Vol. 17, N² 2, 2019, pp. 287-297

https://doi.org/10.22190/FULL1902287V

Review Paper

\title{
LA RENAISSANCE ET LE CLASSICISME : PARALLÈLES ENTRE DEUX ÉPOQUES DES LETTRES FRANÇAISES
}

\author{
UDC 821.133.1.02
}

\section{Nermin Vučelj}

Faculté de philosophie, Université de Niš, Serbie

\begin{abstract}
Résumé : La pierre angulaire de la Renaissance française et de l'Âge classique français fait leur enracinement dans l'antiquité gréco-romaine. Si l'humanisme refonde l'héritage antique et le Grand Siècle le reprend, devrait-il y voir une simple continuité entre l'époque de l'humanisme et l'Âge classique? Parmi les théoriciens, les uns considèrent le classicisme français comme un second humanisme ou nomment la Pléiade la première école du classicisme, voire ils accentuent les parallélismes soulignant les similitudes entre ces deux époques des lettres françaises. Les autres théoriciens signalent la rupture du classicisme avec l'époque précédente et accentuent l'opposition entre la réforme de la Pléiade et la langue ronsardienne de l'imagination débridée, d'une part, et, d'autre part, la doctrine classique et la langue malherbienne annonçant la clarté et le rationalisme du Grand Siècle. Pour susciter de nouvelles considérations à ce sujet, cette recherche revalorise des parallèles entre deux époques et leurs poétiques que les théoriciens ont fait dans l'histoire littéraire, de la fin du XIX siècle, tout au long $d u X X^{e}$ siècle, jusqu'au début du XXI siècle.
\end{abstract}

Mots-clés : Renaissance, humanisme, classicisme, le XVI siècle, le XVII siècle, antiquité gréco-romaine.

\section{REPENSER LA RENAISSANCE FRANÇAISE ET L'ÂGE CLASSIQUE FRANÇAIS}

Lieu commun de toutes histoire et théorie de la littérature font leurs périodisations des courants poétiques et de la production littéraire selon lesquelles, au nom d'une connaissance raisonnée voire systématisée, on répartit l'ensemble des lettres en époques dont chacune d'elles se lie à un siècle historique. C'est la raison pour laquelle, selon Alain Viala (2014, 183), «nous avons l'habitude de confondre dans une même représentation mentale » une époque littéraire et un siècle de calendrier au détriment de la vérité historique qui est plus

Submitted July 2, 2019; Accepted September 17, 2019

Corresponding author: Nermin Vučelj

University of Niš, Faculty of Philosophy

E-mail: nerminvucelj@gmail.com 
complexe. Le $\mathrm{XVI}^{\mathrm{e}}$ siècle égale la Renaissance, le classicisme est le meilleur synonyme du $\mathrm{XVII}^{\mathrm{e}}$ siècle, le XVIII ${ }^{\mathrm{e}}$ se nomme celui des Lumières. Bien que ces termes d'emblées désignant les siècles littéraires soient arbitraires, les théories et histoires de la littérature ne renoncent guère à cette sorte de généralisation, en risquant ainsi de ne donner qu'un tableau simplifié et partiel sur une période des lettres au lieu d'instaurer un regard approfondi et juste.

Toute étude philologique du passé affronte un obstacle difficile à surmonter: on considère une période passée du point de vue de sa propre époque. Toujours est-il que la compréhension d'une ancienne culture est façonnée par notre cadre culturel. Nos interprétations sont un regard à présent sur un esprit d'autrefois ? Suffit-il de juger d'une ancienne période culturelle par nos critères actuels ou faut-il restituer le passé même en saisissant l'esprit de l'époque dans son cadre originel ?

\subsection{Question de terminologie}

Communément, les appellations des périodes particulières viennent bien plus tard - tels que la Renaissance, le baroque, le classicisme - et n'existaient pas dans les époques auxquelles ils ont été attribués a posteriori. Rappelons-nous avec Daniel Ménager qu'au début du XIX ${ }^{\mathrm{e}}$ siècle apparaît l'idée que la Renaissance forme une période étant «l'aurore des temps modernes » et « le réveil des études » suscité par la « renaissance de l'antiquité », et qu'on invente le mot humanisme, provenant de l'allemand Humanismus, pour désigner «le mouvement intellectuel associé au réveil des langues et de la littérature anciennes » (Ménager 1968, 33-34). ${ }^{1}$ C'est l'avènement du romantisme français qui impose le terme de classicisme, obtenu par la substantivation de l'adjectif « classique », auquel on a attribué une connotation négative, celle d'un académisme sclérosé et de la poétique dépassée étouffant l'enthousiasme créateur. Néanmoins, les partisans des valeurs classiques ont approprié le terme de classicisme en tant que notion de suprématie culturelle du Grand Siècle.

Alors que les termes humanisme, Renaissance, classicisme avaient été inventés au XIX ${ }^{\mathrm{e}}$ siècle, rappelons-nous avec Alain Génetiot que celui de classique avait été bien attesté au $\mathrm{XVII}^{\mathrm{e}}$ siècle dont la définition renvoyait « exclusivement aux auteurs de l'Antiquité posés en modèles de perfection littéraire » (Génetiot 2005, 9). Suivons l'étymologie de l'adjectif « classique » avec Michel Delon (Delon 2006, 23) : du latin classici cives, signifiant « les citoyens de la première classe et les plus grosses fortunes de la cité », on vient aux classici testes désignant « les témoins irréprochables, dans un glissement des valeurs sociales aux valeurs morales », pour finir avec autores classici - «comme des écrivains de premier plan », en faisant ainsi le passage de signification des valeurs morales aux valeurs esthétiques. En dehors de la littérature latine, le terme de classique s'applique de même aux bons auteurs des XVII ${ }^{\mathrm{e}}$ et XVIII ${ }^{\mathrm{e}}$ siècles, ce qui Dumarsais avait déjà noté dans son article paru au troisième tome de l'Encyclopédie de Diderot et de d'Alembert. ${ }^{2}$

\footnotetext{
${ }^{1}$ Le terme de Renaissance en tant qu'époque et non plus pour désigner un renouveau des lettres et des arts, a été utilisé pour la première fois en 1840 par l'historien Jean-Jacques Ampère, puis par Jules Michelet en 1855. Le terme Humanismus a été utilisé pour la première fois en 1808 par Friedrich Immanuel Niethammer pour désigner les études de l'antiquité gréco-romaine, et ce n'était qu'en 1841 que ce terme commence à signifier l'entreprise humaniste de Quattrocento, l'époque de la première Renaissance en Italie au XV $\mathrm{XV}^{\mathrm{e}}$ siècle, et cette notion de la découverte de l'héritage grec et latin et de la renaissance des sciences et des arts sera largement acceptée dès 1859. (Giustiniani 1985, 172).

${ }^{2}$ Le cadre n'est plus celui de l'enseignement des collèges, mais de la normalisation linguistique et esthétique, constante Michel Delon concluant que l'article de Dumarsais est «traversé par une tension entre un modèle
} 


\subsection{Question de méthodologie}

Étant donné que les notions de Renaissance, humanisme et classicisme avaient été inventés a posteriori, bien après les périodes auxquelles on a donné ces noms, l'enjeu de notre contemporanéité, des critères actuels, de ce «à présent », joue évidemment un rôle primordial dans nos études de l'esprit d'une époque pédagogiquement encadrée par des dates arbitraires. $\mathrm{Vu}$ sous cet angle, les questions suivantes s'imposent : nos recherches ne font-elles que simplifier l'image du passé dans le noble dessein de l'éclaircir (le XVI ${ }^{\mathrm{e}}$ siècle égale la Renaissance, le XVII ${ }^{\mathrm{e}}$ siècle égale le classicisme) ; nos considérations des époques étudiées restent-elles encrées dans des hypothèses issues de notre temps, au lieu de s'affirmer comme des catégories que les auteurs d'un passé qui est en question approuveraient et que notre postérité confirmerait? L'objectivité philologique est-elle une illusion vaniteuse ? Ou c'est la relativisation du tout, qu'Alan Sokal et Jean Bricmont (Sokal et Bricmont 1997, 33) nomment « un relativisme cognitif et culturel » envahissant les études humaines du dernier quart du $\mathrm{XX}^{\mathrm{e}}$ siècle, qui réduit toute étude humaniste, $\mathrm{y}$ compris des études littéraires, à des narrations ou constructions partielles parmi d'autres, ce qui est la conséquence du rejet du rationalisme hérité des XVII ${ }^{\mathrm{e}}$ et $\mathrm{XVIII}{ }^{\mathrm{e}}$ siècles. Les deux époques des lettres françaises figurant dans le titre de cette recherche contestent par leur principe majeur que les critères de la recherche et de la réception, du jugement et de l'observation critique sont instaurés par la raison, ce qui devrait assurer leur intemporalité.

Cette recherche tente de revaloriser des parallèles entre deux périodes des lettres françaises et leurs poétiques - la première époque, celle de l'humanisme redécouvrant l'antiquité qu'elle prend pour le mentor ; la seconde époque, celle du classicisme appliquant les leçons des Anciens dans le contexte français. Leur rapport à l'antiquité fait le point de départ dans cet article. Tout d'abord, une note concernant la terminologie. L'humanisme et la Renaissance sont habituellement pris en synonyme, et dans cette recherche, qui est relative à la Renaissance française ou l'humanisme français, le terme de Renaissance ou d'humanisme est utilisé en visant toujours les lettres françaises. En ce qui concerne la seconde époque, l'appellation Âge classique français, le convenable d'ailleurs, se croise ici avec le terme de classicisme désignant une poétique et sa production littéraire qui marquent une période de la culture nationale, ce qui nous permet d'utiliser tous les deux, l'Âge classique et le classicisme, en synonyme pour le Grand siècle des lettres françaises. ${ }^{3}$

\subsection{La première école classique et la seconde renaissance}

Le dénominateur commun de deux époques faisant objet de cette recherche est leur enracinement dans l'antiquité gréco-romaine. Si l'on instaure le principe de l'imitation comme le critère de distinction des courants littéraires, en faisant la différence entre deux pistes de l'inspiration poétique, dont la première serait la théorie de l'imitation des

ancien et une norme récente », voire « entre deux définitions de ce qui ne se nomme pas encore le classicisme » (Delon 2006, 24).

${ }^{3}$ Il n'est pas sans importance de nous rappeler deux approches terminologiques : d'une part - celle de la théorie allemande et anglo-saxonne utilisant la notion de néoclassicisme pour désigner les mouvements néoclassiques dans les littératures nationales modernes, selon laquelle le vrai classicisme n'est que celui de l'antiquité gréco-romaine ; d'autre part - la piste critique de la théorie française qualifiant le classicisme français comme spécifique et caractéristique de la culture française. Alain Génetiot souligne que « qualifier le classicisme français de néoclassicisme reviendrait [...] à l'insérer dans une série de doubles dégradés d'un classicisme antique idéal et originel à parité avec d'autres moments classiques nationaux qui ne sont pas, bien souvent, les fleurons de leurs littératures respectives », mais ce qui n'est pas le cas du classicisme français. (Génetiot 2005, 2). 
Anciens et la seconde la théorie de l'imitation de leurs immédiats devanciers, selon Génetiot, «l'âge classique pourrait recouvrir toute la période qui va de la Renaissance au romantisme, de Du Bellay à Lamartine inclus » (Génetiot 2005, 62). Les présupposés théoriques remontent à la Pléiade et «le moment classique [est] ainsi préparé depuis la Renaissance » $(2005,69)$, ce qui fait du classicisme « un second humanisme », qui se caractérise à la fois «par la concentration historique de chefs-d'œuvre comme en une seconde Renaissance » et «par une aspiration à la perfection du modèle antique qui accorde, à la manière d'Horace, les règles avec le goût naturel » (Génetiot 2005, 69).

La thèse de Génetiot selon laquelle «l'âge classique pourrait recouvrir toute la période qui va de la Renaissance au romantisme » correspond à la thèse de René Bray sur l'ère classique commençant avec la Pléiade, dominant le XVII ${ }^{\mathrm{e}}$ siècle et se prolongeant à travers le XVIII ${ }^{\mathrm{e}}$ siècle. René Bray (Bray 1963, I) dégage les trois caractéristiques de la littérature classique : son principe - qui est l'imitation de l'antiquité introduite en France par la Pléiade, sa doctrine - qui est l'ensemble des règles régissant cette imitation, son goût - qui fait triompher l'idéal classique dans l'exécution des œuvres. Autour de ces trois idées Bray esquisse l'évolution de l'esthétique classique en trois étapes qui sont marquées par Ronsard, Chapelain, Boileau, ces trois noms représentant les trois principes - imitation, règle, goût. Bray $(1963,364)$ trace ainsi trois périodes de l'ère classique : la première commençant avec le manifeste de Du Bellay et qui finit avec le $\mathrm{XVI}^{\mathrm{e}}$ siècle dans laquelle l'imitation des Anciens est instaurée ; la deuxième inaugurée par Malherbe et dominée par Chapelain, et qui prend fin vers 1660 - dans laquelle la doctrine est instaurée ; la troisième commençant avec les critiques de Boileau et qui se prolonge à travers le XVIII ${ }^{\mathrm{e}}$ siècle - dans laquelle le goût classique est instauré.

\section{LES POINTS DE RUPTURE ENTRE LE CLASSICISME ET LA RENAISSANCE}

Si l'humanisme refonde l'héritage antique et le Grand Siècle le reprend, il ne faut pas supposer qu'il y a une simple continuité entre l'époque de l'humanisme et l'Âge classique. Comme le théoricien soviétique Mokulski (Стефан Стефанович Мокульский) affirme «le classicisme français du XVII ${ }^{\mathrm{e}}$ siècle n'est pas uniquement le prolongement de la Renaissance française, mais aussi son antithèse spécifique » (« Француский классицисм XVII в. явился потому не только продолжением литературы французского Ренессанса, но и своебразной ее антитезой », Анисимов et al. 1946, 337). Le XVII et le $\mathrm{XVI}^{\mathrm{e}}$ siècle s'opposent plus souvent qu'ils ne s'accordent, conclut René Bray $(1963,25)$, car, si nous regardons le détail, les différences entre la Renaissance et le classicisme grandissent et les ressemblances s'estompent $(1963,22)$.

\subsection{Des cabinets doctes aux salons mondains}

Alain Génetiot suit la transition qui va du pédantisme savant des humanistes aux savoirs mondains des honnêtes hommes. La fin du $\mathrm{XVI}^{\mathrm{e}}$ siècle est marquée par la naissance de la poésie mondaine rejetant l'érudition docte. L'expression poétique devient plus euphonique. Les lettres tendent à la simplicité et à la clarté. Ces deux traits caractéristiques de la littérature mondaine, le refus du pédantisme et l'avènement du langage poétique, seront de même ceux du futur classicisme, conclut Génetiot $(2005,63)$. La littérature devient «un art d'agrément » pour une société de loisir raffinée, ce qui marque la rupture avec l'humanisme «jugé trop érudit, obscur et grossier» (Génetiot 
2005, 65). On établit «un nouvel humanisme» qui est plus conforme à la civilité nouvelle de l'âge de l'honnête homme. Les salons prennent place des académies du siècle précédent. Alors que les académies de la Renaissance prétendaient à la haute culture grecque et latine, les salons mondains de l'Âge classique prêchent «la science du monde » s'opposant au « modèle scolaire du pédant» (Génetiot 2005, 177). Étant érudit, spécialiste et philologue, voire encyclopédiste, humaniste appartient au $\mathrm{XVI}^{\mathrm{e}}$ siècle et ne communique son savoir qu'entre ses homologues.

Selon Antoine Adam, les salons de la première moitié du XVII ${ }^{\mathrm{e}}$ siècle «voulaient seulement rapprocher quelques beaux esprits et des gens du grand monde » (Adam 1968, 31). Grâce aux salons, on fait sortir les lettres des académies humanistes pour les rendre publiquement utiles. La littérature progressiste du XVII ${ }^{\mathrm{e}}$ siècle (nous devons ce terme et la conclusion qui suit à Mokulski) quitte l'universalité et l'encyclopédisme de la Renaissance. Génetiot nomme ce phénomène «la mondanisation des lettres » (2005, 129) : la doctrine devient civile, à la fois laïque et polie ; les lettres et les savoirs deviennent sociaux et communicationnels. Dans ce contexte, le classicisme peut se définir comme « la traduction civile des grands problèmes d'esthétique littéraire posés par l'humanisme » (Génetiot 2005, 126). ${ }^{4}$ De l'âge de l'éloquence, qui est celui de la Renaissance, on passe à l'âge de la conversation, celui du classicisme $(2005,156)$.

\subsection{De l'imitation formelle à l'imitation libérale}

Bien que René Bray ait esquissé les trois étapes de l'évolution de l'esthétique classique autour de ces trois noms - Ronsard, Chapelain, Boileau, en attribuant à chacun d'eux un principe esthétique imitation, règle, goût, l'ère classique, que ce dix-septiémiste français voit sur la ligne temporelle qui va de la Pléiade au romantisme, n'est pas unique et uniforme. Bien que les deux siècles allassent dans le même sens, le second était allé beaucoup plus loin que le premier et «s'est affirmé à peu près hors de toute influence » de la Renaissance (Bray 1963, 22). Le XVII ${ }^{\mathrm{e}}$ siècle ne continue pas le XVI ${ }^{\mathrm{e}}$ siècle. La Renaissance est «un essai de classicisme dont le véritable classicisme est historiquement indépendant» (Bray 1963, 22). L'imitation des Anciens commence avec la Renaissance, affirme Bray, mais la Renaissance s'en tient à l'imitation formelle. Le XVII ${ }^{\mathrm{e}}$ siècle se détourne de l'imitation formelle pour proclamer l'imitation libérale, à savoir les droits de l'invention (1963, 162-163). Les classiques condamnent Ronsard pour avoir suivi de trop près ses modèles - constate Bray. L'instauration de la doctrine des règles par Chapelain et l'instauration du goût par Boileau ont été tracées par Malherbe qui avait rompu avec Ronsard. Or, l'œuvre essentielle de Malherbe n'est pas son cuvre positive mais son cuvre négative: «en combattant Ronsard, il a rendu possible un renouvellement nécessaire », conslut Bray $(1963,8)$. Donc, les deux siècles s'opposent lorsque deux conceptions s'affrontent, représentées par le duel Ronsard - Malherbe. Bray conclut que c'est ainsi que le $\mathrm{XVII}{ }^{\mathrm{e}}$ siècle a rompu avec le $\mathrm{XVI}^{\mathrm{e}}$ siècle.

Antoine Adam affirme qu'il est « trop simple de prétendre » que le XVII ${ }^{\mathrm{e}}$ siècle a été unanime à mépriser « la poésie de notre Pléiade d'inspiration humaniste » (Adam 1968, 97). Bien au contraire - «la tradition humaniste reste si vivante à travers la première moitié du XVII ${ }^{\mathrm{e}}$ siècle », et les théoriciens du classicisme, Chapelain, Balzac, Ménage, en se trouvant dans des cercles d'érudits tout remplis des littératures grecque et latine, ont pris des préoccupations et des habitudes des philologues. Il faut préciser qu'Adam ne voit pas la Cour et des salons mondains comme les seuls acteurs donnant le ton à la littérature de l'époque,

\footnotetext{
${ }^{4}$ Pour Génetiot le classicisme est « une littérature de vulgarisation des motifs et des questionnements de la littérature savante, agités autrefois par les clercs et les doctes et maintenant rendus accessibles au public mondain, celui des gens de cour et des dames » $(2005,125)$.
} 
mais il y compte aussi la bourgeoisie cultivée, « qui ne joue pas un moindre rôle » (Adam $1968,96)$, et qui est attachée à la tradition humaniste beaucoup plus que l'aristocratie.

L'Université, le Parlement de Paris et la bourgeoisie admirait Ronsard. Néanmoins, la dernière édition complète de Ronsard paraît en 1630. Désormais, les critiques sont plus nombreuses que les éloges et on reproche à la poésie ronsardienne « l'imitation servile des Anciens et la négligence dans le style », comme le note Bray résumant la réception de ce prince des poètes de la Renaissance dans les phrases suivantes (1963, 22) : «L'influence de Ronsard disparait dès 1600. Sa réputation, ébranlée par Malherbe, décline entre 1630 et 1660. Le mépris vient après 1660. L'oubli ne tardera pas longtemps. »..$^{5}$ En dépit des admirateurs de Ronsard dans la première moitié du XVII ${ }^{\mathrm{e}}$ siècle, Adam affirme que, à la différence de la Renaissance, la société française du Grand Siècle était « trop nettement orienté vers l'avenir pour s'enfermer dans l'imitation des littératures anciennes » $(1968,29)$.

\subsection{Du latin au français}

Le XVII ${ }^{\mathrm{e}}$ siècle a condamné ce que le manifeste de la Brigade, à savoir de la Pléiade, a proclamé : le français ouvert accueillant le lexique du grec, du latin, de l'italien, des parlers de province et le vocabulaire des artisans. Cette « démesure » est mal vue, remarque Adam $(1968,79)$; désormais, la langue gagne « en s'appauvrissant, donc en se purifiant » (1968, 81). Les classiques donnent la suprématie au français auquel, semble-t-il, les humanistes préfèrent le latin. Yves Giraud et Marc-René Jung affirment que « la littérature en langue vulgaire demeure en dehors de la bataille humaniste », car les humanistes méprisent la langue maternelle et écrivent en latin : « dès qu'ils se servent de leur langue maternelle, comme Rabelais ou Calvin, ils s'écartent de l'humanisme ou s'y opposent même » (Giraud et Jung 1972, 63).

L'approche critique de Daniel Ménager est bien opposée à celle de Giraud et Jung. Il ne considère pas comme de vrais humanistes «les émules de Cicéron» et «les écumeurs du latin » (Ménager 1968, 31), mais ceux qui se déclarent partisans du français, comme Rabelais. De même, le prestige de la langue française est-il attesté par le manifeste de Du Bellay dont le titre est bien significatif - Défense et illustration de la langue française (1549). Mokulski se trouve à la ligne des considérations théoriques de Ménager. Selon ce philologue soviétique, les auteurs qui écrivent exclusivement en latin et imitent servilement les Anciens sont «les humanistes au sens restreint»(Анисимов et al. 1946, 212). Ils renoncent à la contemporanéité vivante et tentent de se plonger mentalement dans le passé antique. Pour «les humanistes au sens large » l'antiquité représente une source importante mais auxiliaire, et non pas obligatoire dans le procès de la création des idéaux culturels et moraux qui sont tout contemporains. Le devoir des humanistes est de comprendre correctement la nature humaine.

\subsection{De l'hellénisme à la latinité}

L'adoration de l'antiquité fait l'un des moments majeurs qui unit le classicisme et la Renaissance (« Преклонение перед античным искусством было одним из наиболее сушественных моментов, связывавших классицизм с искусством Ренессанса », Анисимов et al. 1946, 348) - affirme Mokulski et continue que la compréhension de l'antiquité chez les classiques avait un caractère abstrait et rationaliste. En outre, les

${ }^{5}$ En 1693, Boileau constate que Ronsard, Du Bellay et Desportes ne trouvent même pas de lecteurs (Bray 1963, 21). 
classiques français sont orientés vers l'art grec moins que vers l'art latin en trouvant celui-ci plus froid, plus sévère, et moins attaché à la tradition du peuple. L'antiquité républicaine, présente chez Corneille, a été remplacé par le césarisme romain. De même, René Bray note que «le XVI ${ }^{\mathrm{e}}$ siècle va plus volontiers aux Grecs, le XVII ${ }^{\mathrm{e}}$ aux Latins » (Bray 1963, 25). Roy C. Knight souligne aussi cette différence : le XVII ${ }^{\mathrm{e}}$ siècle n'offre qu' « une synthèse simplifiée et latinisée » du monde grec, mais «n'a jamais compris, ou peut-être s'est peu soucié de comprendre, les différences profondes qui distinguent les civilisations » (Knight 1974, 17). Le XVI ${ }^{\mathrm{e}}$ siècle était donc l'époque la plus brillante de l'hellénisme français, et non pas le XVII ${ }^{\mathrm{e}}$ siècle, conclut Knight. L'honnête homme du $\mathrm{XVII}^{\mathrm{e}}$ siècle a connu la Grèce de seconde main, privé de contacts direct car il ne savait pas le grec ancien. Le XVII ${ }^{\mathrm{e}}$ siècle ne lit plus et semble avoir oublié les ouvrages de l'époque précédente. C'est pourquoi en 1686 on peut prétendre que Platon n'est pas encore traduit en français, comme Knight explique, et note $(1974,41-43)$ : les premiers traducteurs du philosophe grec paraissent dans l'entourage de Marguerite de Navarre ; le Grand Siècle possédait presque tous les prosateurs grecs grâce aux traducteurs de l'époque de la Renaissance ; l'humanisme a légué au classicisme Plutarque, Héliodore, Hérodote, Thucydide, Xénophon, Polybe traduits, ensuite les trois versions complètes d'Homère. Quant au théâtre grec, alors qu'au XVI ${ }^{\mathrm{e}}$ siècle quatre tragédies avaient été traduites, « le XVII ${ }^{\mathrm{e}}$ siècle l'a connu encore moins par la traduction que par des adaptations dramatiques » $(1974,54)$.

Knight ne voit aucun des poètes et penseurs du XVII ${ }^{\mathrm{e}}$ siècle qui serait en premier lieu un disciple des Grecs, et, selon ce théoricien, l'Âge classique marque, à ce point de vue, « un temps d'arrêt». Le climat intellectuel du temps a permis à des hommes sans culture classique de passer pour des honnêtes gens, des beaux esprits, des hommes de lettres, poètes ou philosophes, ce que Knight voit comme un «phénomène nouveau depuis la diffusion des connaissances par l'imprimerie » $(1974,133){ }^{6}$ Knight nomme les hellénistes du XVII ${ }^{\mathrm{e}}$ siècle qui ne savaient pas le grec ou le connaissaient mal $(1974,35,38,39)$ : Ménage n'a jamais lu aucun auteur grec sans avoir lu la traduction latine ; Lefebvre avoue qu'il porte « un si mince bagage d'helléniste »; La Bruyère, qui s'est imposé, en tant que traducteur de Théophraste, comme helléniste et défenseur des Anciens, a traduit son auteur grec en lisant le latin et le contrôlant par un rapide examen du texte grec correspondant ; La Fontaine lisait Homère, Platon et Plutarque en latin.

De même, Knight note que le terme de helléniste avait une connotation négative pour les jésuites, une reproche grave faite aux messieurs du Port-Royal qui témoignent «de l'affectation et du zèle indiscret en faveur de la langue grecque au préjudice de la latine » ce qui mène directement « à la ruine des langues latine et française » $(1974,31)$. Knight considère les jansénistes comme les seuls maîtres du $\mathrm{XVII}^{\mathrm{e}}$ siècle » qui ont fait bien connaître à leurs élèves l'antiquité païenne $(1974,32)$. Il n'est pas étonnant lorsque ce théoricien conclut que Jean Racine, étant disciple de Port-Royal, est le seul vrai helléniste du XVII ${ }^{\mathrm{e}}$ siècle $(1974,134)$.

\footnotetext{
${ }^{6}$ Antoine Adam constate que la culture latine du XVII ${ }^{\mathrm{e}}$ siècle ne domine pas la vie culturelle des Français, qu'elle ne sert qu'à orner de beaux esprits en quoi l'Âge classique se diffère de l'époque de Guillaume Budé et de Jean Dorat marquée par la profonde connaissance des chefs-d'œuvre grecs et latins. Selon Adam l'aristocratie cultivée du temps de Louis XIII, qui méconnait le grec, ne possédait pas la culture des grandes dames de la Renaissance (Adam 1968, 29, 31).
} 


\section{L'HUMANISME DE DEUX SIÈCLES}

Mokulski souligne (1946, 345) que les fondateurs du classicisme ont mené la guerre contre l'individualisme hérité de la Renaissance, celui qui s'exprime comme le culte d'un subjectivisme arbitraire à travers l'inspiration désordonnée. La purification de la langue est exécutée au nom d'une organisation raisonnée de l'expression poétique. Tout comme Descartes a proclamé la raison comme le juge suprême pour le vrai, Chapelain et d'Aubignac l'ont proclamé au même titre pour le beau. Le théoricien soviétique (1946, 345) affirme que Le Discours de la méthode a largement influencé la noblesse et la bourgeoisie progressistes, en leur apprenant comment réduire les choses et les hommes à l'essentiel et en les incitant à bannir toute liberté du domaine de la pensée. La logique cartésienne, développée par les solitaires du Port-Royal, devient le principe de la littérature classique - conclut Mokulski. Le classicisme français s'instaure ainsi comme l'équivalent artistique du rationalisme cartésien.

\subsection{La fougue de la Renaissance et la discipline du classicisme}

De sa part, Antoine Adam proclame que «c'est par une erreur étrange que nous avons pris l'habitude de lier la pensée et la littérature du XVII ${ }^{\mathrm{e}}$ siècle à la philosophie cartésienne » (1968, 70). L'honnête homme «pense selon les formes du gassendisme », à savoir : il est prudent, méthodique, tout occupé des leçons de l'expérience ; et il craint les vues systématiques. Adam affirme que le gassendisme a inspiré la théorie de la doctrine classique de Chapelain et celle de Vaugelas sur la langue et la grammaire. Lorsque Vaugelas déclare que l'usage est le seul maître de la langue, son roi et son tyran, c'est bel et bien la doctrine de Gassendi appliquée à la grammaire - explique Adam (1968, 70). Alors qu'Adam affirme que le classicisme n'a rien avec le cartésianisme mais avec le gassendisme, Génetiot $(2005,167)$ ne reconnaît ni l'influence de Gassendi ni celle de Descartes sur la poétique classique : le cartésianisme reste dans des milieux spécialisés jusqu'en 1660, et il n'avait été diffusé qu'après cette date par l'intermédiaire des jansénistes du Port-Royal ; quant à l'œuvre de Gassendi, écrite en latin, elle restait inaccessible pour un public plus large.

Quelle influence était prépondérante sur la poétique du classicisme, celle du cartésianisme ou celle du gassendisme, c'est la question qui oppose les théoriciens littéraires. Néanmoins, ils semblent être unanimes à propos de la transformation qui mène de la Renaissance au classicisme. Toutes ces considérations similaires peuvent se résumer par les mots de Gustave Lanson. Le XVII ${ }^{\mathrm{e}}$ siècle recueille les résultats de la grande agitation du $\mathrm{XVI}^{\mathrm{e}}$ siècle et celui-là se dégage du celui-ci : « la fougue cède à la discipline, la sensibilité à la raison, le lyrisme à l'éloquence » (Lanson 1912, 228). Michel Foucault développe cette thèse dans son étude Les mots et les choses (1966). Selon lui, au $\mathrm{XVI}^{\mathrm{e}}$ siècle les choses et les mots désignant les choses correspondaient parfaitement, ils se ressemblaient. «Chercher le sens, c'est mettre au jour ce qui se ressemble»- explique Foucault $(2008,44)$. La nature des choses ne se diffère pas de leur ressemblance. On fait la connaissance du monde en suivant les chemins de la similitude. On soulève les signes pour regarder ce qu'ils désignent, et il y apparaît " dans sa propre lumière la Ressemblance elle-même» (Foucault 2008, 44). La similitude au XVI ${ }^{\mathrm{e}}$ siècle triomphait donc de l'espace et du temps: «car il appartenait au signe de ramener et de réunir » $(2008,74)$, de découvrir un contenu caché. Le langage de la Renaissance devient celui du commentaire. 
À partir du XVII ${ }^{\mathrm{e}}$ siècle « le langage se déploie à l'intérieur de la représentation » et il n'a plus d'autre valeur qu'en elle. Il devient discours. Il a la valeur démonstrative - c'est de l'art de «faire signe », de signifier une chose, de nommer ; il a la valeur décorative c'est l'art de disposer de ce signe, de ce nom, de le désigner par d'autres noms qui en sont «le signe second, la figure, l'apparat rhétorique » (Foucault 2008, 58). Le signe apparaît comme le résultat de l'analyse : «parce que l'esprit analyse, le signe apparaît » (2008, 75). Ainsi la raison occidentale entre-t-elle dans «l'âge du jugement», celui du classicisme qui analyse la représentation, à savoir il analyse comment un signe peut être lié à ce qu'il signifie $(2008,58)$.

Alors que le langage du XVI ${ }^{\mathrm{e}}$ siècle était «dans une posture de perpétuel commentaire », au $\mathrm{XVII}^{\mathrm{e}}$ siècle il devient la critique qui s'occupe de la représentation. Au lieu de la ressemblance, nous avons la représentation ; au lieu du commentaire, ce qui était le langage de la Renaissance, dans le classicisme «tout langage valait comme discours », en tant qu'analyse critique : et «la critique ne peut analyser le langage qu'en termes de vérité d'exactitude, de propriété ou de valeur expressive » (Foucault 2008, 94). À l'énigme d'une parole qu'un second langage doit interpréter (ce qui est le commentaire dans la Renaissance), s'est substituée la discursivité essentielle de la représentation (ce qui est la critique dans l'Âge classique), ce qui se résume dans cette formulation de Foucault : «[...] le commentaire a fait place à la critique » $(2008,94)$.

\subsection{L'humanisme au sens synchronique}

Dans leur collection littéraire, utilisée durant des décennies au lycée français pour former des esprits cultivés, mais aujourd'hui complètement bannie des écoles françaises, sans être remplacée par un meilleur manuel dans l'enseignement qui, semble-t-il, a aussi renoncé à cultiver des esprits, Lagarde et Michard nous rappelle que «ce beau terme d'humanitas » évoque « une élégance morale, une politesse, une courtoisie, inséparables de toute culture accomplie » (Lagarde et Michard 1985, 8). L'humanisme est tout ce qui fait un homme vraiment homme, c'est un acte de foi dans la nature humaine. Cette compréhension du concept humaniste dépasse la notion originelle, celle de la formation à l'école de la pensée gréco-latine. L'humanisme devient ainsi, restons avec Lagarde et Michard dans leur tome consacré au XVI ${ }^{\mathrm{e}}$ siècle, « un idéal de la sagesse et de toute une philosophie ». Toutes ces significations sont relatives au classicisme, ainsi qu'à la Renaissance. Dans leur tome consacré au XVII ${ }^{\mathrm{e}}$ siècle, ces co-auteurs de l'anthologie et l'histoire littéraires considèrent le classicisme comme un humanisme (Lagarde et Michard 2004, 10) : le dénominateur commun des classiques et des humanistes fait l'analyse de la nature humaine et l'art et les lettres sont considérés à l'échelle de l'homme. Selon Lagarde et Michard $(1985,12)$ la Renaissance française commence comme «un énorme appétit de savoir» et «un optimisme sans bornes»; sa deuxième phase est sous le signe de l'art défini comme l'imitation des Anciens, succédée par la phase du «goût plus raffiné » et d'« un idéal de perfection formelle » annonçant le classicisme.

Gustave Lanson reconnaît le lien qui unit l'Âge classique à l'époque de l'humanisme français et conclut que «sous le pédantisme de la Renaissance commence à percer l'originalité classique » (Lanson 1912, 359). Si nous suivons la définition selon laquelle l'époque de la Renaissance française représente «l'histoire de l'introduction de l'idée d'art et de son adaptation à l'esprit français » (Lanson 1912, 222), cette approche nous mène à voir dans le classicisme français l'idéal accompli, la perfection réalisée, le sublime 
saisi. Il semble que les convergences entre les deux époques, la Renaissance française et l'Âge classique français mettent en lumière l'évolution d'un humanisme pris au sens synchronique - à savoir comme un état d'esprit, une école éthique et une conception esthétique, dont le point de départ est la Renaissance et le point d'arrivée le classicisme.

Si nous dépassons le cadre des lettres françaises, l'humanisme apparaît comme la notion de périodes dans lesquelles la civilisation saisit le plus haut niveau culturel dont le centre et le but fait l'homme. Le premier humanisme que nous connaissons dans le monde occidental est l'antiquité grecque, la culture latine fait le deuxième humanisme, le troisième est celui de l'Italie de Quattrocento, suit la Renaissance française, et l'Âge classique dont la place prendra l'humanisme du siècle philosophique. Les deux premières décennies du XXI siècle souffrent de l'absence des lumières de la raison. Désorientée à cause de la relativité du tout qui la domine, la contemporanéité en 2019 ne reconnaît point de bon goût et ne croit plus en valeur impérissable. Insensible, déshumanisée et robotisée, la civilisation d'aujourd'hui a désespérément besoin d'une renaissance de l'humanité.

Note : Cet article est rédigé dans le cadre du projet scientifique Les langues, les littératures et les cultures romanes et slaves en contact et en divergence, $N^{o}$ 81/1-17-8-01, approuvé par la Faculté de Philosophie de l'Université de Niš et soutenu par l'Agence universitaire de la Francophonie.

\section{RÉFÉRENCES BIBLIOGRAPHIQUE}

Анисимов, Иван Иванович, Стефан Стефанович Мокульский и Алекса́ндр Алекса́ндрович Смирно́в. История франиузской литературы, том 1, С древнейших времен до Революиии 1789 г. Москва Ленинград, Академии Наук СССР, 1946.

Adam, Antoine. L'Âge classique - I, 1624-1660. Littérature française, t. VI. Paris, Arthaud, 1968.

Bray, René. La Formation de la Doctrine Classique en France. Paris, Librairie Nizet, 1963.

Delon, Michel. «Classicismes, Lumières et romantisme », dans Jean-Charles Darmon, Michel Delon (dir.), Histoire de la France littéraire. Paris, Presses Universitaires de France, 2006, p. 23-36.

Foucault, Michel. Les mots et les choses. $1^{\text {ère }}$ éd. 1966. Paris, Gallimard, 2008.

Génetiot, Alain. Le classicisme. Paris, Presses Universitaires de France, 2005.

Giraud, Yves, et Marc-René Jung. La Renaissance - I, 1480-1548. Littérature française, t. III. Paris, Arthaud, 1972.

Giustiniani, Vito R. «Homo, Humanus, and the Meanings of Humanism ». Journal of the History of Ideas, 46 (2), 1985, p. 167-195.

Knight, Roy C. Racine et la Grèce. Paris, Librairie A.-G. Nizet, 1974.

Lagarde, André, et Laurent Michard. XVI siècle - les grands auteurs français. Paris, Bordas, 1985.

Lagarde, André, et Laurent Michard. XVII e siècle - les grands auteurs français. Paris, Bordas, 2004.

Lanson, Gustave. Histoire de la littérature française. Paris, Hachette, 1912.

Ménager, Daniel. Introduction à la vie littéraire du XVI siècle. Paris, Bordas, 1968.

Sokal, Alan, et Jean Bricmont. Impostures intellectuelles. Paris : Édition Odile Jacob, 1997.

Viala, Alain. Une histoire brève de la littérature française - Le Moyen Âge et la Renaissance. Paris, Presses Universitaires de France, 2014.

Viala, Alain. Une histoire brève de la littérature française - L’Âge classique et les Lumières. Paris, Presses Universitaires de France, 2015. 


\section{RENESANSA I KLASICIZAM: PARALELE IZMEĐU DVE EPOHE U FRANCUSKOJ KNJIŽEVNOSTI}

Ugaoni kamen francuske renesanse i francuske klasične epohe čini njihova ukorenjenost $u$ grčko-rimsku antiku. Ako humanizam obnavlja antičko nasleđe, a francusko klasična doba ga preuzima, možemo li između humanizma i klasicizma videti tek jednostavni kontinuitet? Među književnim teoretičarima $i$ istoričarima, jedni gledaju na francuski klasicizam kao na drugi humanizam ili nazivaju Plejadu prvom školom klasicizma, tj. ističu paralele kojima se podvlače sličnosti između dve epohe u francuskom umetničkom stvaralaštvu. Prema drugim teoretičarima, klasicizam raskida s prethodnom epohom tako da su međusobno suprotstavljenje, s jedne strane, Plejadina reforma i ronsarovski jezik koji je u znaku neobuzdane mašte, $i$, s druge strane, klasična doktrina $i$ malerbovski jezik koji najavljuje jasnost $i$ racionalizam klasične epohe. U nameri da podstakne nova razmatranja na ovu temu, ovo istraživanje nastoji da preispita pristupe književnih teoretičara koji su upoređivali dve epohe i njihove poetike - francusku renesansu XVI veka $i$ francuski klasicizam XVII veka.

Ključne reči: renesansa, humanizam, klasicizam, XVI vek, XVII vek, grčko-rimska antika 